D. L. Ashley - M. A. Bonin - B. Hamar

M. McGeehin

\title{
Using the blood concentration of 2,5-dimethylfuran as a marker for smoking
}

Received: 4 December 1994/Accepted: 2 August 1995

\begin{abstract}
Correct analysis of whole blood volatile organic compounds (VOCs) in evaluating possible exposure situations requires differentiation of smokers from nonsmokers. Whole blood concentrations of 2,5-dimethylfuran are determined using an internal standard method, and the concentrations of this compound are evaluated as a marker for smoking in exposure-study subjects. Results indicate that the concentration of 2,5dimethylfuran can be adequately determined in whole blood by a method already in use for determining VOCs in blood. The whole blood concentration of 2,5-dimethylfuran was an excellent predictor of smoking when compared with positive responses about smoking on questionnaires. Using a detection limit of $0.024 \mathrm{ppb}, 2,5$-dimethylfuran concentrations in blood correctly identified the smoking status of $96.4 \%$ of the subjects in this study. The blood 2,5-dimethylfuran concentration was linearly related to the number of cigarettes smoked per day. This method is advantageous since blood 2,5-dimethylfuran concentrations can be determined using the same method used to determine concentrations of other VOCs, thus obviating the need for additional analytical procedures.
\end{abstract}

Key words Volatile organic compounds · 2,5-Dimethylfuran

D. L. Ashley $(\mathbb{\Omega}) \cdot$ M. A. Bonin

Division of Environmental Health Laboratory Sciences,

National Center for Environmental Health,

Centers for Disease Control and Prevention,

4770 Buford Highway, Mailstop F-17, Atlanta,

GA 30341-3724, USA

B. Hamar · M. McGeehin

Health Investigations Branch, Division of Health Studies, Agency for Toxic Substances and Disease Registry, Atlanta, GA 30333, USA

\section{Introduction}

The science of epidemiology depends on the ability of investigators to sort through the abundance of available information and accurately characterize those risk factors that are related to a disease or other health condition. Error in the classification of these risk factors can confound this analysis by either diluting the responsible relationships so that they are underestimated or falsely indicating relationships that do not actually exist. Much of the information required for epidemiologic analysis is obtained by personal interviews and questions submitted to the people involved. In many cases this is the only way to obtain this information.

The use of questionnaires is subject to a wide variety of problems, including limited recall, questioner bias, and falsification. Falsification is particularly prevalent where the questions involve certain moral or social issues such as alcohol consumption, sexual activity, and drug use. With the increased controversy concerning cigarette smoking and other tobacco use, using questionnaires to evaluate smoking status and frequency is also becoming more difficult. When possible, actual analytical determinations that give quantitative findings are preferred in place of questionnaires.

To interpret the measurements of volatile organic compounds (VOCs) we need to be able to distinguish smokers from nonsmokers. Previous papers have reported that the levels of benzene, toluene, and other VOCs in breath and blood are affected by cigarette smoking (Angerer et al. 1991; Brugnone et al. 1989; Perbellini et al. 1988; Wallace et al. 1987; Wang et al. 1993). In exposure studies a different true prevalence of cigarette smoking between comparison groups can confound the analysis or increase the variance, overwhelming any small disparity between these groups.

Currently the best method a vailable for determining exposure to tobacco smoke is measuring cotinine in 
serum or urine (Cummings et al. 1990). This method appears to give excellent agreement with smoking status, but using urinary cotinine measurements to evaluate VOC results requires collecting an additional sample and performing a separate analysis.

In 1990 an investigator (Gordon 1990) examined smokers' breath in an attempt to identify a component that could be used as a definitive marker of smoking. $\mathrm{He}$ found that of the 230 gas chromatography/mass spectrometry peaks present in breath, 2,5-dimethylfuran was the most indicative of smoking status. In these studies he showed that 2,5-dimethylfuran was not present in any nonsmokers' breath but could be found in the breath of $92 \%$ of the smokers examined. Since this compound in breath was highly associated with smoking, we examined whether it could be detected in blood using our current VOC analysis method, and whether blood concentrations could be used as a marker to distinguish smokers from nonsmokers.

\section{Materials and methods}

The method used for measuring VOCs in human blood has been described elsewhere (Ashley et al. 1992). The procedure used for this study is a modification of this method to include 2,5-dimethylfuran as an analyte. A summary of the key details and particular modifications for the measuring 2,5-dimethylfuran are given here.

\section{Safety}

Many of the chemical materials used in this study are considered toxic. For others the toxicity has not been clearly established. We carried out all manipulations in accordance with accepted practice for safe handling of toxic chemicals. Since blood samples were not prescreened for hepatitis B or the human immunodeficiency virus, we performed all sample handling under a biological safety cabinet with appropriate personal protection, including gloves, gown, and mist respirator. All laboratory equipment that came in contact with blood samples was decontaminated by using a $10 \%$ sodium hypochlorite solution.

\section{Materials and apparatus}

We obtained 2,5-dimethylfuran and benzene- ${ }^{13} \mathrm{C}_{6}$ from Aldrich Chemical (Milwaukee, Wisc.) and Cambridge Isotope Laboratories (Wobum, Mass.), respectively. We acquired purge-and-trap grade methanol from Burdick \& Jackson (Muskegon, Mich.).

The purge and trap apparatus consisted of a Tekmar (Cincinnati, Ohio) LSC 2000 purge and trap concentrator with an attached ALS 2016 automated sampler. Chromatography was carried out in a Hewlett-Packard (Avondale, Penns.) Model 5890 gas chromatograph with the column passing through a heated interface directly into the mass spectrometer ion source. The chromatograph was equipped with a J\&W (Folsom, Calif.) 30-m DB-624 column with a $1.8-\mu \mathrm{m}$ film thickness. The helium flow rate was maintained at $30 \mathrm{ml} / \mathrm{min}$ at $20 \mathrm{psi}$. We used Fisons (Danbury, Mass.) $7070 \mathrm{E}$ or $70 \mathrm{~S}$ high-resolution mass spectrometers operating at 3000 resolving power for detection, identification, and quantification. Experimental measurements showed equivalent results from these two instruments.
Mass values were referenced to perfluorokerosene. The instrument was operated in full-scan mode $(40-200 \mathrm{amu})$ with a scan rate of $1.0 \mathrm{~s} /$ decade and a settling time of $0.2 \mathrm{~s}$.

Procedures

Samples were collected as part of a health and exposure assessment carried out by the Agency for Toxic Substances and Disease Registry. The study population included 100 persons residing near a hazardous waste site area and 106 persons who were frequency matched by sex and age and had been selected from a comparison community. We administered questionnaires to each person after obtaining his or her informed consent to participate in the study. We did not ask questions about the smoking status of children younger than 12 years old, and their blood samples were removed from further analysis. In addition to other health-related questions, we asked about recent VOC exposure scenarios, alcohol consumption, and smoking history. We based our determination of smoking status on a person's response to the question, "Do you smoke cigarettes now?"

Two $10-\mathrm{ml}$ blood samples were collected by venipuncture into vacutainers (Becton Dickinson Vacutainer Systems, Rutherford, N.J.) containing a mixture of potassium oxalate and sodium fluoride. These vacutainers had been treated previously to remove VOC contaminants and analyzed to verify their removal (Ashley et al. 1992). Samples were stored at refrigerator temperatures $\left(4^{\circ} \mathrm{C}\right)$, and measurements were carried out within 7 weeks of sample collection. Previous studies have shown that blood levels of most VOCs do not change substantially over this period (Ashley et al. 1994). After we introduced antifoam B emulsion (Sigma Chemical, St. Louis, Mo.) into the sample spargers, we decontaminated them overnight by heating and helium purging. We added $20 \mu \mathrm{l}$ of a stable isotope analog solution to $10-\mathrm{ml}$ blood samples after extracting the samples through the vacutainer caps with an airtight syringe. The entire syringe contents were injected into the sampling spargers. We then directly purged the blood samples to remove the VOCs.

Quantification was achieved by monitoring mass $96.06 \pm 0.03$ amu for 2,5-dimethylfuran and mass $84.07 \pm 0.03 \mathrm{amu}$ for benzene${ }^{13} \mathrm{C}_{6}$. We used standard calibration curves of response factor divided by sample weight versus standard concentration to calculate unknown concentrations. The identity of the analyte was verified by examining retention times and full-scan spectra and comparing these times and spectra to those of standard compounds. Blanks, standards, and quality control materials were included in each analytical run to ensure analytical stability, the maintenance of sensitivity, and the absence of contamination. The detection limit was determined by previously described techniques (Taylor 1987) and was $0.024 \mathrm{ppb}$ for 2,5-dimethylfuran.

\section{Results}

Figure 1a shows a typical limited-mass range $(96.06 \pm 0.03)$ chromatogram for 2,5-dimethylfuran in human blood. For this sample 2,5-dimethylfuran is found at scan 688 , which is baseline separated from other components. The full-scan mass spectrum of scan 688 is shown in Fig. 1b. Spectral peaks at masses 96,95 , 81,53 , and 43 amu correspond to the major peaks in the standard 2,5-dimethylfuran spectrum, verifying the identity of this analyte. Additional mass peaks result from other blood components isolated by the purge and trap technique. Separation by mass selective analysis is required to prevent interference from these other components. 

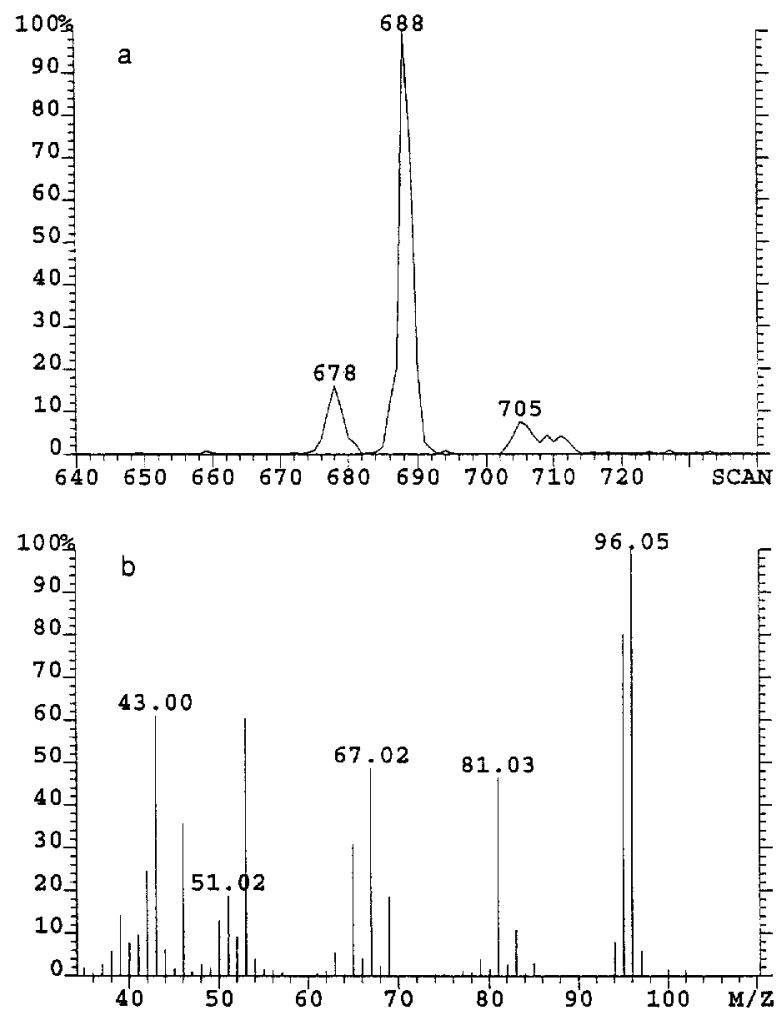

Fig. 1a, b Typical gas chromatographic and mass spectral results for 2,5-dimethylfuran in whole blood. a Accurate mass chromatogram $(96.06 \pm 0.03 \mathrm{amu})$. b Mass spectrum of scan 688

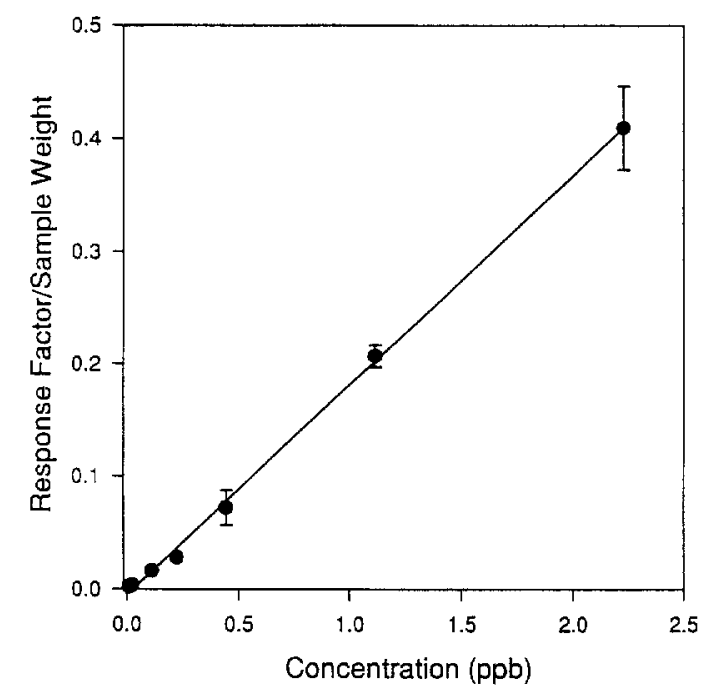

Fig. 2 Standard curve (response/sample weight versus concentration) for 2,5-dimethylfuran with linear least-squares fit of data. Error bars, $95 \%$ confidence limits for each standard examined

Figure 2 shows a typical calibration curve (five replicates at each standard concentration) for dimethylfuran along with the best-fit linear least-squares regression curve. The $R^{2}$ for this fit was 0.97 , and the
Table 1 Summary results for 2,5-dimethylfuran levels (ppb) in whole blood separated by smoking response for case, comparison, and combined populations

\begin{tabular}{|c|c|c|c|c|c|}
\hline \multirow[t]{2}{*}{ Population } & \multicolumn{2}{|c|}{ Reported nonsmokers } & \multicolumn{2}{|c|}{ Reported smokers } & \multirow[t]{2}{*}{$P$} \\
\hline & Mean $(n)$ & Range & Mean $(n)$ & Range & \\
\hline Case & $\mathrm{ND}^{\mathrm{a}}$ & $N^{a}-0.13$ & $0.14(19)$ & $0.044-0.32$ & 0.0001 \\
\hline Comparison & $\mathrm{ND}^{\mathrm{a}}(62)$ & $\mathrm{ND}^{\mathrm{a}}-0.055$ & $0.14(23)$ & $\mathrm{ND}^{\mathrm{a}}-0.28$ & 0.0001 \\
\hline Combined & $\mathrm{ND}^{\mathrm{a}}(127)$ & $\mathrm{ND}^{\mathrm{a}}-0.13$ & $0.14(42)$ & $N^{a}-0.32$ & 0.0001 \\
\hline
\end{tabular}

${ }^{a}$ Results below limit of detection $(0.024 \mathrm{ppb})$

curve was linear over two orders of magnitude from 0.024 to $2.2 \mathrm{ppb}$. All detectable 2,5-dimethylfuran results in whole blood were found within this range. Under some circumstances this curve appeared to be nonlinear and required a second-order least-squares fit to properly characterize the calibration data.

Accuracy and precision were examined by spiking whole blood samples with solutions made by diluting neat 2,5-dimethylfuran and measuring the subsequent concentration. Five repeat measurements were performed at each spiking level of 0.1 and $0.5 \mathrm{ppb}$. Combining these data showed that 2,5-dimethylfuran mean recovery using this method was $97.8 \%$, and the coefficient of variation was $10.9 \%$. These results are similar to those previously reported by us for other volatile chemicals (Ashley et al. 1992).

We distinguished smokers from nonsmokers on the basis of their response to an inquiry about whether they currently smoked. Even though we asked questions about previous smoking history, only the responses about whether people currently smoked were used as a basis for establishing an individual's smoking status. Table 1 summarizes results of measuring 2,5-dimethylfuran, separated by the smoking-questionnaire response for the case, comparison, and combined-subject populations. Results of the Student's $t$ test analysis comparing smokers and nonsmokers are also given. In all cases, there was a statistically significant difference between the blood 2,5-dimethylfuran concentrations for smokers and nonsmokers.

Figure 3 shows the frequency distribution of the concentration of 2,5-dimethylfuran in the blood of smokers and nonsmokers. These results clearly indicate that 2,5-dimethylfuran is related to smoking as characterized by individual questionnaire responses. Separating blood 2,5-dimethylfuran concentrations into detectable and nondetectable levels and comparing these levels with smoking status produces the information shown in Table 2. These data indicate that the sensitivity of this method using reported smoking status as the standard for comparison is $95 \%$, and the specificity is $97 \%$. This analysis demonstrates the effectiveness of using 2,5-diemethylfuran levels in whole blood as a marker for smoking. For this study 2,5dimethylfuran concentrations in whole blood, using 


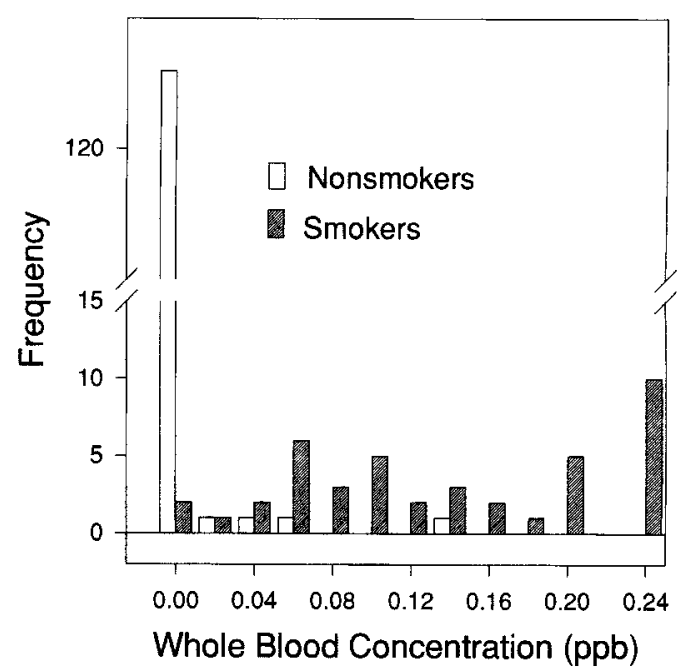

Fig. 3 Frequency distribution of concentration of 2,5-dimethylfuran in blood of smokers $(n=42)$ and nonsmokers $(n=127)$

Table 2 Smoking questionnaire response and 2,5-dimethylfuran detectability from all whole blood samples collected $(D L$, detection limit $0.024 \mathrm{ppb})$

\begin{tabular}{lll}
\hline & $\begin{array}{l}\text { Reported smoking } \\
(n=42)\end{array}$ & $\begin{array}{l}\text { Reported nonsmoking } \\
(n=127)\end{array}$ \\
\hline $\begin{array}{lll}\text { Dimethylfuran > DL } & 40 & 4 \\
\text { Dimethylfuran < DL } & 2 & 123\end{array}$ \\
\hline
\end{tabular}

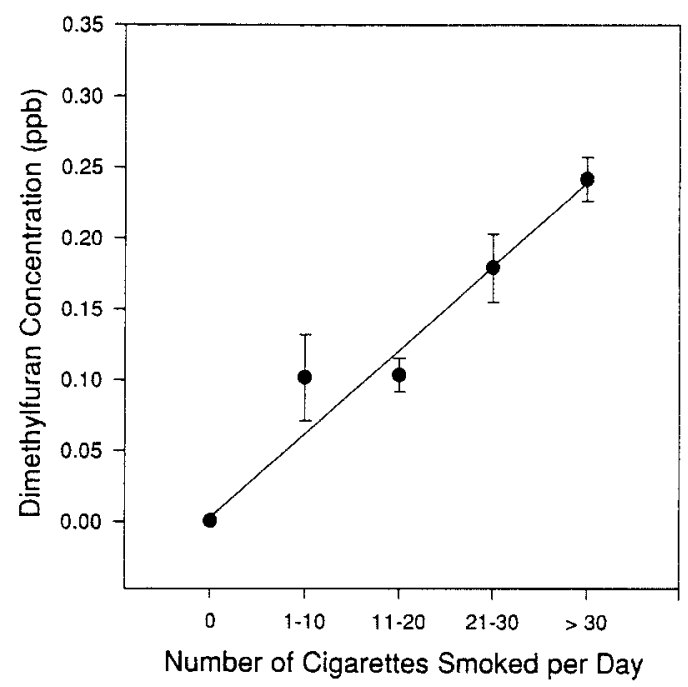

Fig. 4 Plot of the mean 2,5-dimethylfuran concentration (ppb) versus cigarettes smoked per day. Number of cigarettes are grouped as shown. Error bars, standard error of the mean for each cigarette group

a cutoff equal to the detection limit, had a $4.8 \%$ false-negative rate and a $3.1 \%$ false-positive rate, so that $96.4 \%$ of the respondents were correctly classified on the basis of questionnaire responses.
An additional questionnaire response concerned the number of cigarettes each person estimated that he or she smoked each day. Grouping their responses into ranges of $0,1-10,11-20,21-30$, and greater than 30 cigarettes, we found that the number of cigarettes smoked is linearly related to the 2,5-dimethylfuran concentration found in whole blood $\left(R^{2}=0.74\right.$ $P=0.0001$ ). This relationship is shown in Fig. 4. Analysis using the specific number of cigarettes smoked per day indicates that each cigarette contributes approximately $0.006 \mathrm{ppb}$ to the blood, 2,5-dimethylfuran concentration.

\section{Discussion}

Our results clearly show that 2,5-dimethylfuran can be effectively measured in whole blood by techniques previously used for measuring VOCs. Using an internal standard method, we were able to achieve sufficient detection limits, recovery, and reproducibility to differentiate smokers from nonsmokers. It is likely that using a labeled analog in place of the internal standard method will improve analytical parameters and should be investigated.

A comparison of 2,5-dimethylfuran levels with questionnaire responses shows that the whole blood concentration of 2,5-dimethylfuran is an effective tool for differentiating smokers from nonsmokers. In addition, these results demonstrate that 2,5-dimethylfuran concentrations in whole blood can be used to estimate the number of cigarettes that a person smokes each day.

Previous studies have shown that 2,5-dimethylfuran is present in tobacco smoke. Baggett et al. (1974) and Higgins et al. (1983) examined cigarette smoke and found many VOCs including benzene, toluene, xylenes, and styrene in addition to 2,5-dimethylfuran. The level of 2,5-dimethylfuran, along with the other VOCs, was generally correlated with tar delivery, suggesting that the blood levels of this compound may not only be dependent on the number of cigarettes but also the type of cigarette smoked. There may be a number of additional smoking-related variables, including the time elapsed since the last cigarette was smoked and the number of cumulative months of smoking, that affect the 2,5-dimethylfuran blood concentration. Other studies are required to differentiate these variables.

There are other possible sources of 2,5-dimethylfuran which must be considered when evaluating the usefulness of the levels of this compound in blood to quantify smoking status. Previous studies (Wang et al. 1983; Shimoda and Shibamoto 1990) have identified 2,5dimethylfuran as a component of the headspace volatiles of brewed coffee. Perbellini et al. (1980) have shown that 2,5-dimethylfuran is a minor metabolite of $n$-hexane. Thus, drinking brewed coffee or exposure to 
high levels of hexane may interfere with using 2,5dimethylfuran as a marker for smoking.

An important use of urinary cotinine measurements has been in analyzing the urine of people exposed to second-hand smoke (Cummings et al. 1990). Preliminary analysis of the results from the present study suggest that 2,5-dimethylfuran levels in whole blood may not be sensitive enough to characterize exposure to second-hand smoke. We characterized exposure to second-hand smoke on the basis of responses to questions about whether the subjects lived or worked with smokers. We found no clear relationship between second-hand smoke exposure and elevated 2,5-dimethylfuran blood concentrations in nonsmokers. The lack of this marker in persons exposed to second-hand smoke is most likely a result of limitations on the sensitivity of the methods used. An alternative cause could be the shorter residence time of 2,5-dimethylfuran in the body than that of cotinine.

As noted above, there are typically some errors when a questionnaire is used to determine confounding factors. Blood concentrations of 2,5-dimethylfuran may actually be a more effective marker for smoking than the results of this study suggest. It is possible that some or all of the four persons who answered that they had not smoked but did have detectable 2,5-dimethylfuran blood concentrations were answering falsely. If this were true, 2,5-dimethylfuran would be an even more effective smoking marker than the present results suggest.

The results for the presence of 2,5-dimethylfuran in blood are very similar to those previously determined for its presence in breath (Gordon 1990). Both studies reveal that 2,5-dimethylfuran is an excellent marker for tobacco smoking. Blood concentrations are particularly suitable for identifying smokers in blood VOC studies, since quantification can be performed by using the same method as for other VOCs. The half-life of 2,5-dimethylfuran in blood must be determined before it is clear whether this analyte is practical in evaluating smoking status. Studies on additional populations are required before blood 2,5-dimethylfuran levels can be used confidently as a biomarker for smoking, but these initial results suggest that this method may be both effective and efficient.

Acknowledgements We wish to acknowledge the contributions of Frederick L. Cardinali, Joan M. McCraw, and Joe V. Wooten to the completion of this study.

\section{References}

Angerer J, Scherer G, Schaller KH, Miller J (1991) The determination of benzene in human blood as an indicator of environmental exposure to volatile aromatic compounds. Fresenius J Anal Chem 339:740-742

Ashley DL, Bonin MA, Cardinali FL, McCraw JM, Holler JS, Needham LL, Patterson DG (1992) Determining volatile organic compounds in human blood in a large sample population by using purge and trap gas chromatography/mass spectrometry. Anal Chem 64:1021-1029

Ashley DL, Bonin MA, Cardinali FL, McCraw JM, Wooten JV, Needham LL (1994) Important considerations in the ultra-trace measurement of volatile organic compounds in blood. In: Mincar RA, Ford AM, Needham LL, Karch NJ (eds) Applications of molecular biology in environmental chemistry. Lewis, Chelsea, pp 135-146

Baggett MS, Morie GP, Simmons MW, Lewis JS (1974) Quantitative determination of semivolatile compounds in cigarette smoke. J Chromatogr 97:79-82

Brugnone F, Perbellini L, Faccini GB, Pasini F, Maranelli G, Romeo L, Gobbi M, Zedde A (1989) Breath and blood levels of benzene, toluene, cumene and styrene in non-occupational exposure. Int Arch Occup Environ Health 61:303-311

Cummings KM, Markello SJ, Mahoney M, Bhargava AK, McElroy PD, Marshall JR (1990) Measurement of current exposure to environmental tobacco smoke. Arch Environ Health 45:74-79

Gordon SM (1990) Identification of exposure markers in smokers' breath. J Chromatogr 511:291-302

Higgins CE, Griest WH, Olerich G (1983) Application of tenax trapping to analysis of gas phase organic compounds in ultralow tar cigarette smoke. J Assoc Off Anal Chem 66:1074-1083

Perbellini L, Brugnone F, Pavan I (1980) Identification of the metabolites on $n$-hexane, cyclohexane, and theor isomers in men's urine. Toxicol Appl Pharmacol 53:220-229

Perbellini L, Faccini GB, Pasini F, Cazzoli F, Pistoia S, Rosellini R, Valsecchi M, Brugnone F (1988) Environmental and occupational exposure to benzene by analysis of breath and blood. $\mathrm{Br}$ J Ind Med 45:345-352

Shimoda M, Shibamoto, T (1990) Isolation and identification of headspace volatiles from brewed coffee with an on-column GC/MS method. J Agric Food Chem 38:802-804

Taylor JK (1987) Quality assurance of chemicals measurements. Lewis, Chelsea, pp. 79-82

Wallace LA, Pellizzari ED, Hartwell TD, Sparacino C, Whitmore R, Sheldon L, Zelon H, Perritt R (1987) The TEAM study: personal exposures to toxic substances in air, drinking water, and breath of 400 residents of New Jersey, North Carolina and North Dakota. Environ Res 43:290-307

Wang G, Maranelli G, Perbellini L, Guglielmi G, Brugnone F (1993) Reference values for blood toluene in the nonocupationally nonexposed general population. Int Arch Occup Environ Health 65:201-203

Wang TH, Shanfield H, Zlatkis (1983) Analysis of trace volatile organic compounds in coffee by headspace concentration and gas chromatography - mass spectrometry. Chromatographia 17:411-417 